wound care and highlighting needed research and education initiatives.

\section{P111 A WORKFLOW CHECKLIST FOR IMPROVING MANAGEMENT OF THE GUIDELINE DEVELOPMENT PROCESS}

${ }^{1,2} \mathrm{M}$ Haller, ${ }^{1,3} \mathrm{E}$ Nagler, ${ }^{1,4} \mathrm{~S}$ van der Veer, ${ }^{1,5}$ I Nistor, ${ }^{1,6} \mathrm{D}$ Bolignano, ${ }^{1,3} \mathrm{~W}$ Van Biesen. ${ }^{1}$ European Renal Best Practice, Ghent University Hospital, Ghent, Belgium; ${ }^{2}$ Center for Medical Statistics (CeMSIIS), Medical University Vienna, Vienna, Austria; ${ }^{3}$ Renal Division, Department of Internal Medicine, Ghent University Hospital, Ghent, Belgium; ${ }^{4}$ Academic Medical Center, Department of Medical Informatics, Amsterdam, The Netherlands; ${ }^{5}$ Nephrology Department, GT. Popa University of Medicine and Pharmacy, Lasi, Romania; ${ }^{6}$ CNR-IBIM, Clinical Epidemiology and Physiopathology of Renal Diseases Reggio, Calabria, Italy

\section{0:1136/bmjqs-2013-002293.166}

Background Keeping track of progress and meeting deadlines can be difficult for organisations that simultaneously develop multiple guidelines involving multiple systematic review teams.

Objectives To improve planning, organising and managing of guideline development projects by creating a standardised workflow checklist.

Methods In a one-day meeting, the five guideline development methodologists, the chairman and the editorial assistant identified the main steps involved in the guideline development process. For each step, we identified specific tasks and ordered them chronologically. All decisions were made based on group consensus. The identified steps and tasks formed the basic elements of the workflow checklist.

Results We identified the need for two separate checklists per guideline development project; one for overall workflow and one for each clinical question covered by the guideline. The overall guideline development workflow checklist comprised 42 tasks organised in 11 sequential steps, including items such as topic selection, composition of the guideline development group, and framing the questions. For each clinical question we identified 27 tasks organised in 8 sequential steps, excluding steps already covered by the overall workflow.

Discussion This workflow checklist represents a first step in developing a standardised project management strategy to improve efficient management of the guideline development process. Further development of this tool involves selecting appropriate software for practical implementation applicable not only for our own means but also for those of other groups.

Implications for Guideline Developers/Users We believe developing a standardised workflow checklist will improve efficient management of guideline development and allows transparent and up-to-date communication of its progress.

\section{P114 THE QUALITY OF EVIDENCE OF SYSTEMATIC REVIEWS OF TRADITIONAL CHINESE MEDICINE: A CROSS- SECTIONAL STUDY}

${ }^{1,2} \mathrm{Y}$ Chen, ${ }^{1,2} \mathrm{Q}$ Wu, ${ }^{1,2} \mathrm{X}$ Wang, ${ }^{1,2} \mathrm{Q}$ Wang, ${ }^{1,2} \mathrm{D}$ Wei, ${ }^{1,2} \mathrm{~L}$ Yao,${ }^{3} \mathrm{~F}$ Liang, ${ }^{1,2}, \mathrm{M}$ Wang, ${ }^{1,2} \mathrm{~K}$ Yang. 'Evidence-Based Medicine Center of Lanzhou University, Lanzhou, China; ${ }^{2}$ Chinese GRADE Center, Lanzhou, China; ${ }^{3}$ The First Hospital of Lanzhou University, Lanzhou, China

\section{0:1136/bmjqs-2013-002293.167}

Background There has been an increasing interest in systematic reviews of traditional Chinese medicine (SR-TCM) over the past
10 years. Little is known about the quality of evidence of SRTCM.

Methods GRADE (Grading of Recommendations Assessment, Development and Evaluation) system is a tool to rate evidence quality of SRs and other evidence body. We searched CBM (China Biomedicine Database) from 1978 to 2012 and included all SR-TCM in the field of cancer treatment. We used GRADE system to assess the quality of evidence of those SRs. Two reviewers independently screened the titles and abstracts of identified studies. Full texts of potentially included articles were further assessed. Disagreements were resolved by discussion.

Results The preliminary results showed that the quality of evidence of SR-TCM were: high (1\%), moderate (25\%), low $(50 \%)$, very low (24\%). We also compared with the quality of evidence of SRs published in Chinese medical journals $(5 \%$, 27\%, 49\%, 19\%) and Cochrane SRs (5\%, 27\%, 49\%, 19\%). Risk of bias, inconsistency and publication bias were the major factors for downgrading evidence of SR-TCM.

Conclusion More and more SR-TCM had been published in Chinese medical journals, however, the proportion of high quality evidence is lower and the very low quality evidence is higher compared with national and international levels.

\section{P115 HOW MANY CLUSTER RANDOMIZED CONTROLLED TRIALS WERE USED IN CLINICAL PRACTICE GUIDELINES?}

${ }^{1,2} \mathrm{Y}$ Chen, ${ }^{1,2} \mathrm{Q}$ Wu, ${ }^{1,2} \mathrm{X}$ Wang, ${ }^{1,2} \mathrm{Q}$ Wang, ${ }^{1,2} \mathrm{D}$ Wei, ${ }^{1,2} \mathrm{~L}$ Yao, ${ }^{1,2} \mathrm{~F}$ Liang, ${ }^{3} \mathrm{M}$ Wang, ${ }^{1,2} \mathrm{~K}$ Yang. 'Evidence-Based Medicine Center of Lanzhou University, Lanzhou, China; ${ }^{2}$ Chinese GRADE Center, Lanzhou, China; ${ }^{3}$ The First Hospital of Lanzhou University, Lanzhou, China

\section{0:1136/bmjqs-2013-002293.168}

Background There has been an increasing interest in cluster randomised controlled trial (CRT) over the past 20 years. Little is known about how many CRTs were used by clinical practice guidelines.

Methods We searched National Guideline Clearinghouse (NGC) which is a public resource for evidence-based clinical practice guidelines on December 31, 2012. We selected guidelines which provide MEDLINE full-text linking and then we checked all references cited by those guidelines.

Results We included 564 guidelines and they contained 57495 references. We identified 13 guidelines cited 17 cluster randomised controlled trials as their references. Guidelines are about primary care, cancer, obesity, breastfeeding, cardiovascular and orthopaedics disease. Screening studies accounts for as much as $35 \%$ (6) of all CRTs.

Conclusion Cluster randomised controlled trial is considered as the golden standard to assess the effect of intervention in health research. Based on the retrieval strategy study for cluster randomised controlled trial we developed, we estimated that there are about 8000 CRTs in Medline, however, only 17 CRTs were used or cited by clinical practice guidelines, the reasons of low utilisation of CRTs in guidelines are now being investigated and we are going to present the final findings.

\section{P117 UNIFORMITY IN ANAESTHESIOLOGY RECOMMENDATIONS}

${ }^{1}$ I Loman, ${ }^{2} \mathrm{~A}$ Schuurhuis. 'Knowledge Institute of Medical Specialists, Utrecht, The Netherlands; ${ }^{2}$ The Netherlands Society of Anesthesiologists, Utrecht, The Netherlands

10:1136/bmjqs-2013-002293.169 